\title{
"Structure from Motion“ zur flächigen Deformationsvermessung unter Tage - Modellversuche als Beitrag zur Entwicklung eines nutzerfreundlichen Messsystems unter Verwendung von Bildern
}

\author{
Magdalena Berger, Alexander Tscharf und Gerhard Mayer \\ Lehrstuhl für Bergbaukunde, Bergtechnik und Bergwirtschaft, Department Mineral Resources Engineering, \\ Montanuniversität Leoben, Leoben, Österreich
}

Eingegangen 24. April 2017; angenommen 25. April 2017; online publiziert 15. Mai 2017

\begin{abstract}
Zusammenfassung: Im vorliegenden Beitrag wurden Modellversuche angestellt, um unter Verwendung moderner digitaler Bildverarbeitung ein benutzerfreundliches Messsystem zur Erfassung von Deformationen im untertägigen Bergbau zu entwickeln. Dazu wurden zwei verschiedene Modelltypen herangezogen - zum einen Betonwürfel, zum anderen leicht deformierbare Tonmodelle, von denen sowohl in undeformiertem als auch in deformiertem Zustand herkömmliche RGB Fotos aufgenommen wurden. Mit Hilfe verschiedener Softwareprodukte wurden aus den Bildern dreidimensionale Rekonstruktionen erstellt und in weiterer Folge miteinander verglichen.

Aus den Ergebnissen geht hervor, dass Deformationen in zeitlich unterschiedlich entstandenen Punktwolken deutlich sichtbar und, je nach Ausmaß, auch messbar sind. Die Versuche zeigen, dass moderne photogrammetrische Verfahren somit durchaus auch in der bergmännischen Praxis anwendbar sind.
\end{abstract}

Schlüsselwörter: Structure from Motion,

Deformationsvermessung, Photogrammetrie

\begin{abstract}
"Structure from Motion" for Deformation Monitoring Underground - Lab-scale Tests to Contribute the Development of a User-friendly Surveying System Using Images
\end{abstract}

Abstract: In the following paper various model tests were carried out in order to develop a user-friendly surveying system appropriate for recording deformations in under-

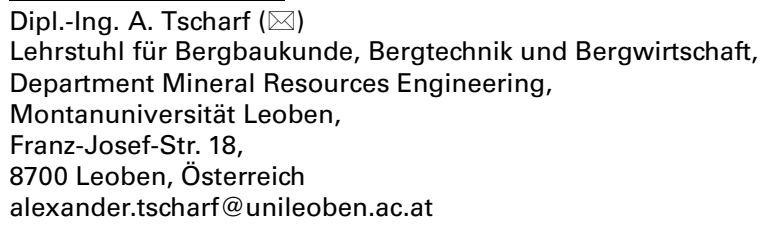

ground mining, using modern digital image processing. For this purpose, two different types of models were examined closely. Firstly, concrete cubes, secondly easily deformable clay models. These clay models were photographed both in their undeformed and in deformed condition. With the help of several software programmes, these conventional RGB photos were transformed into three-dimensional reconstructions and finally compared to one another.

The results show that deformations within variously emerged point clouds are clearly visible and, moreover, measurable. Consequently, these tests demonstrate that photogrammetric processes as such have the potential to be of high relevance within the scope of mining.

Keywords: Structure from Motion, Deformation Monitoring, Photogrammetry

\section{Einleitung und Motivation}

Um Veränderungen frühzeitig zu erkennen und in der Folge entsprechende Maßnahmen einleiten zu können, müssen potentielle Gefahrenbereiche in regelmäßigen Abständen kontrolliert und beobachtet werden. Aus Personal-, Zeitund Kostengründen sind Systeme von Vorteil, die rasch und einfach in der Anwendung sind und somit jederzeit und von allen Mitarbeitern - ohne spezielle Aus- und Vorbildung angewandt werden können. Unter Verwendung handelsüblicher Kameras können sehr schnell und mit geringem Personaleinsatz deformationsgefährdete Bereiche fotografiert und in weiterer Folge zu hochaufgelösten dreidimensionalen Punktwolken prozessiert werden. Ziel wäre es sodann, diese zu unterschiedlichen Zeitpunkten entstandenen Punktwolken miteinander zu vergleichen und womöglich entstandene Deformationen erkennen und quantifizieren zu können. 
Abb. 1: Versuchsaufbau des Betonmodells-undeformierter Zustand

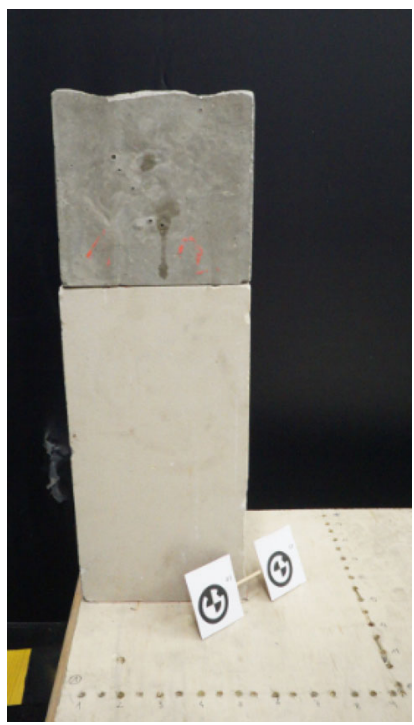

Anhand praktischer Modellversuche sollte untersucht werden, ob es Möglichkeiten gibt, bestehende Bildvermessungssysteme zur einfachen und benutzerfreundlichen Deformationsvermessung unter Tage heranzuziehen. Vordergründig sollten aufwendige Signalisierungsarbeiten vermieden und eine einfache Form zur Skalierung der Ergebnisse entwickelt werden, wodurch die praktische Relevanz des Verfahrens gesteigert werden könnte.

\section{Stand der Technik}

Bei klassischen Verfahren der Geodäsie (Tachymetrie) wird die "theoretisch kürzeste Verbindung sowie die Richtung zwischen zwei Punkten“ [1] gemessen. Diese Messverfahren werden schon seit Langem angewandt und sind aufgrund der elektronischen Distanzmessung sehr genau. Da der Zeitaufwand bei der Vermessung einer großen Anzahl an Einzelpunkten zur detailgetreuen Erfassung des Objekts allerdings sehr groß ist, gewannen in den letzten Jahren flächige und photogrammetrische Messmethoden zunehmend an Bedeutung und stellen heute eine wertvolle Ergänzung (teilweise auch Alternative) zu herkömmlichen Vermessungsmethoden dar.

\subsection{Photogrammetrie}

Mit Hilfe photogrammetrischer Verfahren ist es möglich, aus zweidimensionalen Bildern eines Objektes, im vorliegenden Fall von Modellkörpern, ein möglichst genaues dreidimensionales Bild bzw. virtuelles Modell der Realität abzuleiten [2]. Gemäß dem Modell der Zentralprojektion wird jeder Objektpunkt entlang einer geraden Linie (Bildstrahl) durch das Projektionszentrum am Bildträger abgebildet. Sofern sowohl die innere Orientierung der Kamera als auch die äußere Orientierung - „die Lage des Aufnahmesystems im Raum" - [3] bekannt sind, können die einzelnen Bildstrahlen zu einem 3D-Punkt trianguliert werden (Messung von Richtungen). Man unterscheidet grundsätzlich zwischen der Stereophotogrammetrie, bei der nur zwei Fotos notwendig sind, und der Mehrbildphotogrammetrie, im Zuge derer mehrere Fotos gemacht werden [4]. Jeder Bildstrahl wird vom Objektpunkt, dem Projektionszentrum und dem dazugehörigen Bildpunkt gebildet. Alle Objektpunkte, die zu einem Bildstrahl gehören, führen zum gleichen Bildpunkt. Sobald sich zwei Bildstrahlen von zumindest zwei Bildern schneiden, kann der Objektpunkt im Raum absolut zugeordnet werden [5].

\subsection{Registrierung}

Unter Registrierung wird grundsätzlich das Überführen aller Stand- und Objektpunkte in ein übergeordnetes Koordinatensystem verstanden [6]. Um zwei 3D-Punktwolken aus unterschiedlichen Messepochen miteinander vergleichen zu können, müssen sie ebenfalls in dasselbe Koordinatensystem gebracht werden. Das Einordnen in ein gemeinsames Koordinatensystem bzw. die Schätzung der notwendigen Transformationsparameter kann über verschiedene Wege geschehen, wobei ICP (Iterative Closest Point) von Besel und McKay eine weitverbreitete Methode ist, die weitestgehend automatisiert abläuft und sich auch für die hier beschriebenen Arbeiten am besten bewährt hat.

\section{Modellversuch}

Um herauszufinden, ob es möglich ist, Deformationen bildbasiert und ohne großen Zusatzaufwand zu erfassen, wurden Modellversuche durchgeführt. Das Ziel war, im Modell definierte Deformationen zu erzeugen und anschließend zu überprüfen, ob diese Deformationen mit der bildbasierten Rekonstruktion erfasst werden können. Für die Versuche wurden zwei unterschiedliche Modelltypen verwendet - zum einen zwei Würfel aus Beton, zum anderen ein Modell aus herkömmlichem Töpferton. In den Abb. 1 und 2 ist der undeformierte Zustand, der sogenannte Ausgangszustand, sowohl des Beton- als auch des Tonmodells zu erkennen. Die deformierten Zustände des Betonmodells wurden erreicht, indem der obere Würfel entweder schräg bzw. in eine oder zwei Richtungen verschoben, auf den unteren aufgesetzt wurde. Die "realistischeren" bzw. natürlicheren Deformationen beim Tonmodell wurden händisch erzeugt. Zur Gewährleistung der Reproduzierbarkeit der Versuche, bzw. um die Versuchskörper mehrmals aus der gleichen Kameraposition zu fotografieren, wurde eine kleine Holzpalette mit einem Abmaß von ca. $50 * 80 \mathrm{~cm}$ als "Modelluntergrund" verwendet. Rund um das Modell wurden in regelmäßigen Abständen Löcher gebohrt, von denen jedes eine Kameraposition darstellte. Insgesamt wurden von jeder Kameraposition, in drei verschiedenen Höhen, je zwei Serien der undeformierten als auch der deformierten $\mathrm{Zu}$ stände aufgenommen. 


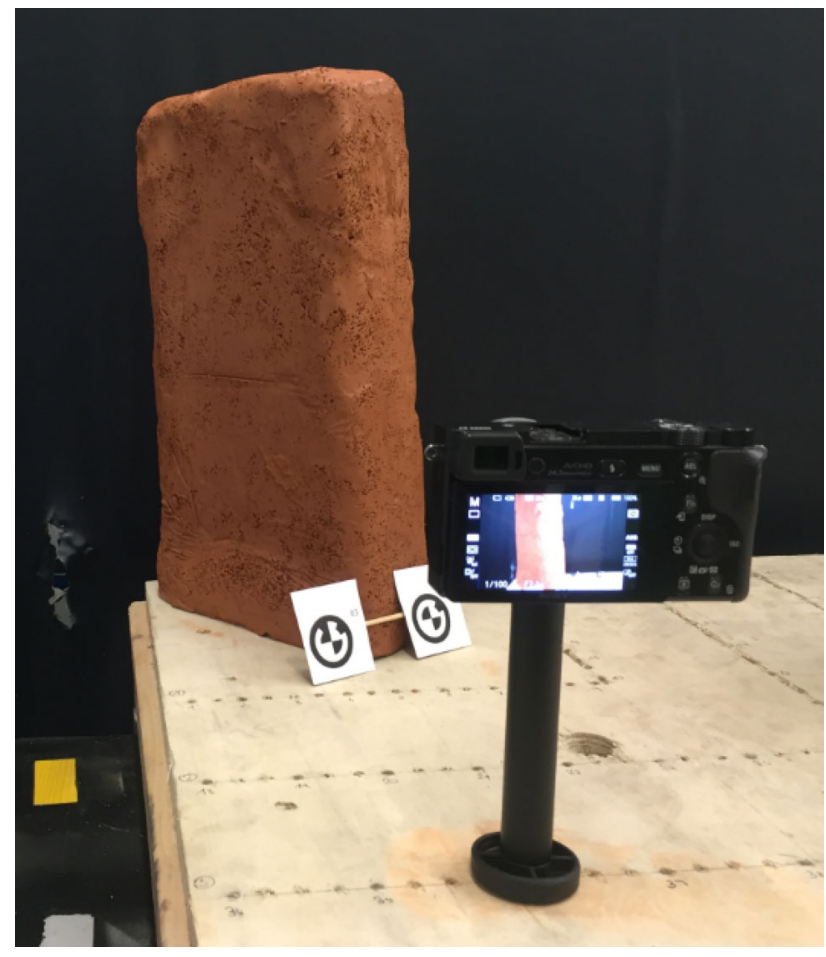

Abb. 2: Versuchsaufbau des Tonmodells-undeformierter Zustand

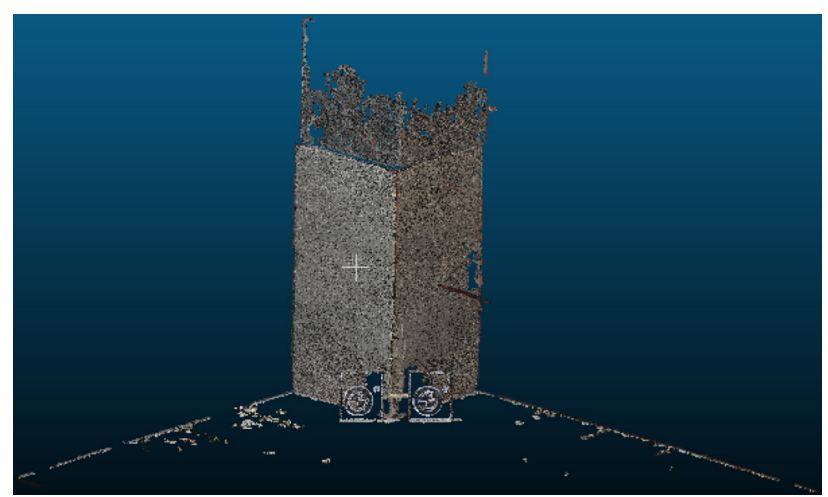

Abb. 3: Dense Wolke

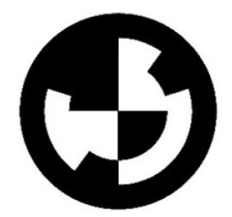

37

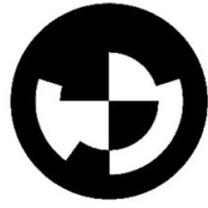

38
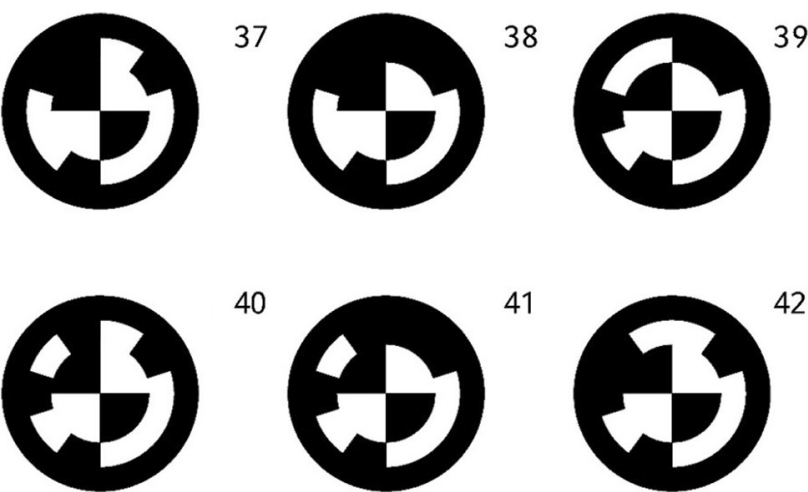

40

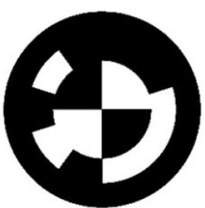

41

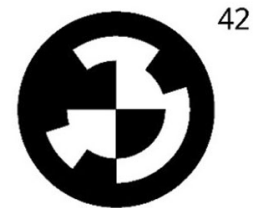

Abb. 4: Marker- „fiducial marker“

\section{Verarbeitung der Daten - Structure from Motion}

Im Gegensatz zur klassischen Photogrammetrie, bei der in zumindest zwei 2D-Bildern, manuell, gleiche Bildpunkte gesucht und markiert werden, ist der Structure from Motion (SfM) Prozess weitgehend automatisiert. Durch die Verwendung moderner Algorithmen (Feature Detection) können bei SfM wesentlich mehr Punkte gefunden werden als bei der klassischen Photogrammetrie, sodass es zu einer deutlichen numerischen Überbestimmung kommt. Pro Bild werden zwischen 10 und 20.000 sogenannte Features gefunden, von denen ungefähr drei- bis fünftausend auch in anderen Bildern wiedergefunden werden können (Matches). Beim Bündelblockausgleich wird jedes Bild durch ein Strahlenbündel dargestellt, die dann hinsichtlich der relativen Orientierung zueinander und basierend auf den Rückprojektionsfehlern der triangulierten 3D-Punkte optimiert werden. Durch die Vielzahl an Bildmessungen können ausschließlich aus der Bildinformation die Koordinaten der 3DObjektpunkte bzw. die Position der Kameras (äußere Orientierung) berechnet werden.

Wichtige Schritte beim SfM-Prozess sind die Kamerakalibrierung, die Merkmalsextraktion, die Korrespondenzanalyse, die relative Orientierung und der Bündelblockausgleich. Bei der Kamerakalibrierung wird mit Hilfe von Markern die innere Orientierung bestimmt [7]. Im nächsten Schritt werden "Natural Features" gefunden [8] und Korrespondenzen zwischen Deskriptoren verschiedener Bilder über die euklidische Distanz berechnet. Bei der relativen Orientierung und dem Bündelblockausgleich wird die Lage der Bilder zueinander und der 3D-Objektpunkte geschätzt bzw. optimiert [9].

\section{1 qt_offline_sfm}

Für die Versuche, die für diese Arbeit relevant waren, wurde die Softwarebibliothek qt_offline_sfm des Instituts für Maschinelles Sehen und Darstellen an der TU Graz verwendet. Auch sie nutzt SfM, um aus den 2D-Bildern 3D-Punktwolken abzuleiten. Die so entstehenden Punktwolken beinhalten ausschließlich die gematchten Features und werden „sparse" Wolken genannt.

\subsection{Densification}

Mittels der Densification werden die wenig verdichteten sparsen Wolken weiter verdichtet, so dass die entstehenden densen Wolken folglich mehr lokale Effekte zeigen und ein viel höher aufgelöstes Bild der Realität wiedergeben (Abb. 3). In dieser Arbeit wurde für die Densification die Patch-based Multi-view Stereo (PMVS) und Clusteringviews for Multi-View Stereo (CMVS) Software von Yasutaka Furukawa verwendet. Der CMVS Preprocessor kachelt große Bilddaten, PMVS dient dann zur Verdichtung von Punktwolken [10]. 
Abb. 5: Registrierung der Punktwolke undefomiert auf die Punktwolke deformiert
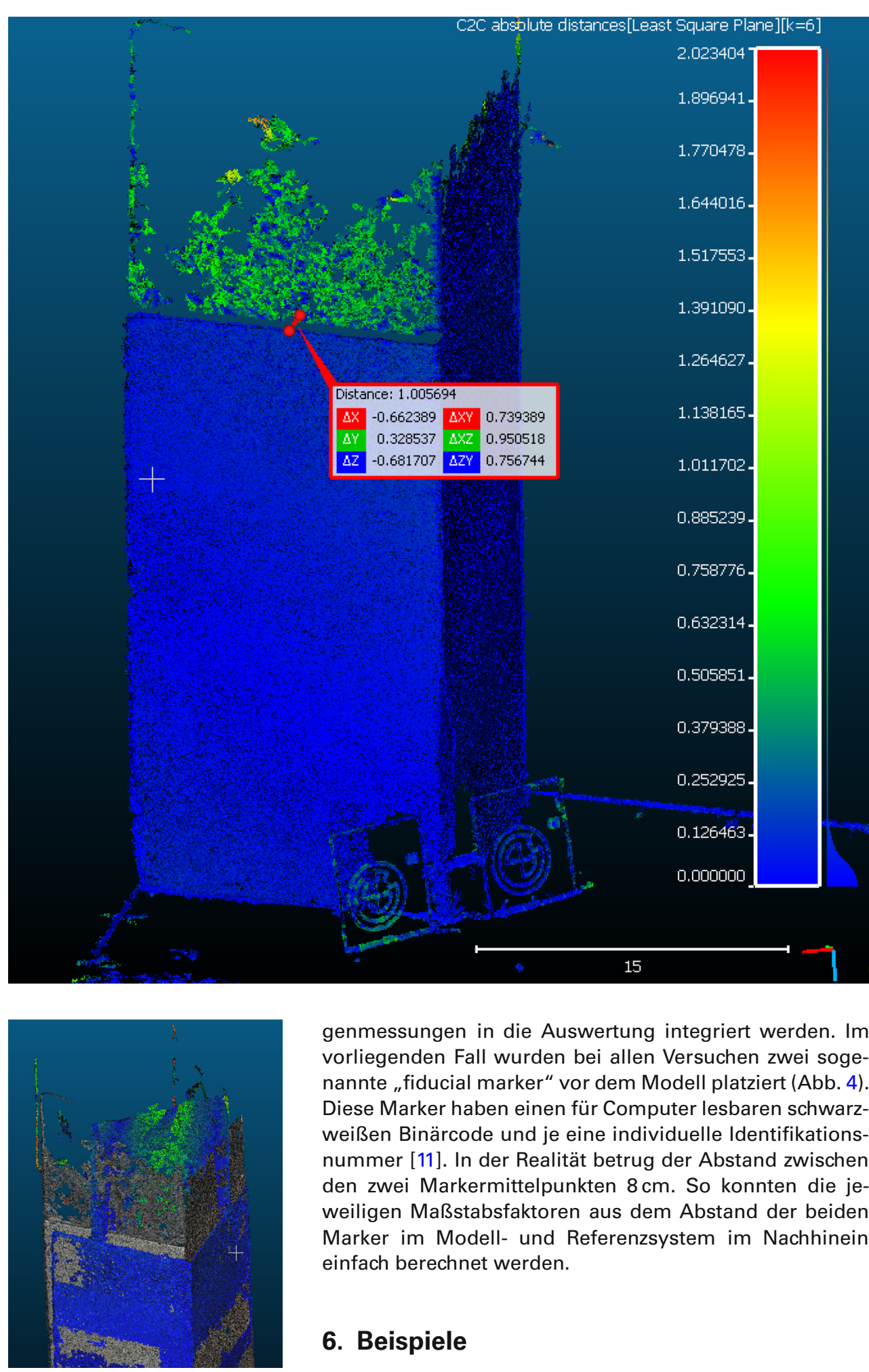

genmessungen in die Auswertung integriert werden. Im vorliegenden Fall wurden bei allen Versuchen zwei sogenannte "fiducial marker" vor dem Modell platziert (Abb. 4). Diese Marker haben einen für Computer lesbaren schwarzweißen Binärcode und je eine individuelle Identifikationsnummer [11]. In der Realität betrug der Abstand zwischen den zwei Markermittelpunkten $8 \mathrm{~cm}$. So konnten die jeweiligen Maßstabsfaktoren aus dem Abstand der beiden Marker im Modell- und Referenzsystem im Nachhinein einfach berechnet werden.

\section{Beispiele}

\subsection{Anhand des Betonmodells}

In Abb. 5 ist die Registrierung der Punktwolke des undeformierten Zustandes auf die Punktwolke eines der deformierten Zustände zu sehen. Im unteren Bereich kann die deutliche Übereinstimmung der beiden Wolken erkannt werden.
Im oberen Bereich wird durch die hellere Färbung eine kan-
Da die rein bildbasierten Rekonstruktionen aufgrund der fehlenden Längenmessung zunächst maßstabsfrei sind und die komplette Auswertung ausschließlich auf dem Messen von Winkeln basiert, müssen zusätzliche Län- 
Abb. 7: Registrierungsbeispiel anhand des Tonmodells

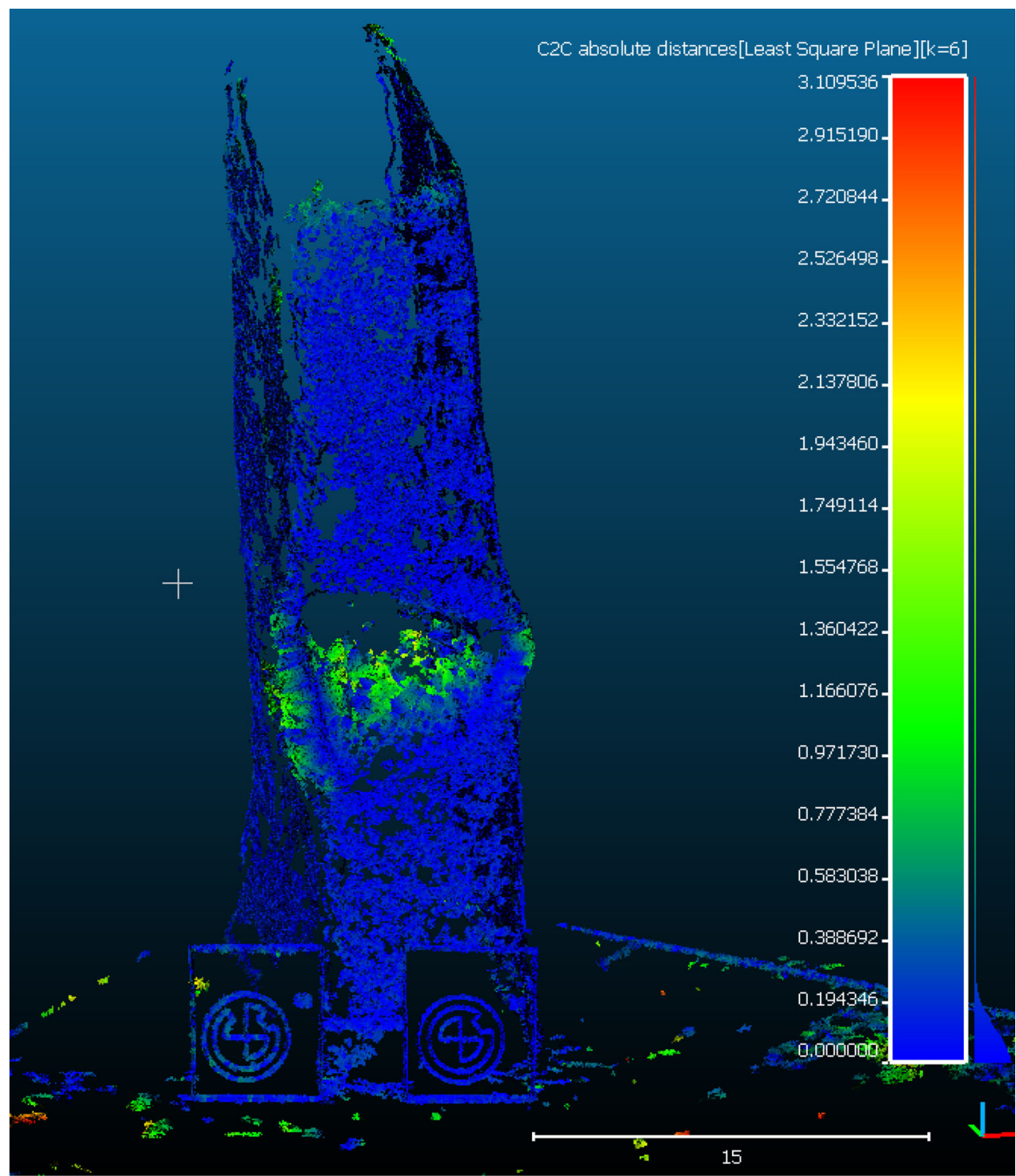

tenparallele Verschiebung des oberen, kleineren Würfels um $1 \mathrm{~cm}$ nach hinten deutlich (Skala Bereich in cm-Angaben).

\subsection{Fehlgeschlagene Registrierung anhand des Betonmodells}

Die Registrierung der Punktwolke des undeformierten Zustandes auf die Punktwolke eines deformierten Zustandes, bei dem der obere Würfel schräg auf den unteren aufgesetzt wurde, zeigte weder in der ersten noch der zweiten Serie wünschenswerte Ergebnisse. Wie in Abb. 6 zu erkennen, sind Deformationen bzw. Verschiebungen sichtbar, jedoch passen weder die Seitenkanten noch sonstige Längen zueinander. Somit konnten Deformationen zwar erkannt, jedoch nicht nachgemessen werden.

\subsection{Registrierung anhand des Tonmodells}

Ebenso wie die Betonwürfel-Versuche, brachten die Tonversuche wünschenswerte Ergebnisse. Wie in Abb. 7 zu sehen, ist stirnseitig eine faustgroße Deformation zu erkennen. Der restliche Bereich ist durchgehend unverändert gegenüber der zweiten undeformierten Wolke.

\section{Ergebnisse und Schlussfolgerung}

Aus den in dieser Arbeit durchgeführten Modellversuchen geht hervor, dass es grundsätzlich möglich ist, unterschiedliche Messepochen rein geometriebasiert aufeinander zu registrieren. Auch bei den „realitätsnahen“ Tonprobekörpern funktionierte die Registrierung und veränderte Bereiche konnten eindeutig ausgemacht werden. Im Gegensatz zu den kantig begrenzten und geometrisch eindeutig beschreibbaren Betonwürfeln ist eine quantitative Erfassung 
von Deformationen bzw. Veränderungen jedoch nur bedingt möglich.

Voraussetzung für das Funktionieren von ICP ist jedoch, dass die Objektgeometrie zumindest ähnlich bleibt und die Deformationen nicht zu groß werden. Das heißt für die Praxis, dass die zeitlichen Abstände zwischen den Messepochen klein genug sein müssen, um Deformationen erkennen bzw. quantitativ messen zu können. Fortschreitende Deformationen müssen regelmäßig fotografiert und in der Folge registriert werden, um deutliche Ergebnisse ableiten zu können. Weiterführende Arbeiten sollen im Detail untersuchen, inwieweit sich Objekte in der Praxis von einer Messepoche zur nächsten verändern bzw. deformieren können, ohne ein Fehlschlagen der Registrierung nach sich zu ziehen. Beachtet werden muss in diesem Fall jedoch, dass die Gesamtdeformation aus den einzelnen Deformationen errechnet werden muss.

Zusammenfassend kann gesagt werden, dass eine grundsätzliche Rekonstruktion aus Bildern und eine Skalierung über den berechneten Maßstab einwandfrei funktioniert. Auch die rein geometriebasierte Registrierung funktionierte innerhalb gewisser Schranken besser als erwartet. Als nächster Schritt könnten Versuche im Realmaßstab durchgeführt, und parallel weitere Möglichkeiten zur räumlichen Registrierung (z. B. unter Verwendung der Bildinformation gemeinsam mit der vorhandenen Geometrie, SLAM) untersucht werden.
Open access funding provided by Montanuniversität Leoben

Open Access Dieser Artikel wird unter der Creative Commons Namensnennung 4.0 International Lizenz (http://creativecommons.org/licenses/ by/4.0/deed.de) veröffentlicht, welche die Nutzung, Vervielfältigung,

Bearbeitung, Verbreitung und Wiedergabe in jeglichem Medium und Format erlaubt, sofern Sie den/die ursprünglichen Autor(en) und die Quelle ordnungsgemäß nennen, einen Link zur Creative Commons Lizenz beifügen und angeben, ob Änderungen vorgenommen wurden.

\section{Literatur}

1. Wikipedia: Geodäsie, https://de.wikipedia.org/wiki/Geod\%C3\%A4sie, (17.02.2017)

2. Luhmann, T.: Nahbereichsphotogrammetrie, Heidelberg: Wichmann, 2000, S. $3 \mathrm{ff}$.

3. Luhmann, T.: Nahbereichsphotogrammetrie, Heidelberg: Wichmann, 2000, S. 8 .

4. Luhmann, T.: Nahbereichsphotogrammetrie, Heidelberg: Wichmann, 2000, S. $8 f$

5. Luhmann, T.: Nahbereichsphotogrammetrie, Heidelberg: Wichmann, 2000, S. 10.

6. Dold, C.; Brenner, C: Verfahren zur Registrierung von 3D Punktwolken: http://www.geomatik-hamburg.de/tls/tls2007/vortraege/ 03 tls2007_dold.pdf, (17.02.2017)

7. Daftry, S.; Maurer, M.; Wendel, A., Bischof, H.: Flexible and Usercentric Camera Calibration Using Planar Fiducial Markers, 2013

8. Lowe, D.: Distinctive Image Features from Scale-invariant Keypoints, International Journal of Computer Vision 60 (2004), iss.2, p. $91-110$

9. Triggs, B.; Mc Lauchlan, P.; Hartley, R.; Fitzgibbon, A.: Bundle adjustment - A modern synthesis, 2000

10. Pomaska, G.: 3D-Objekt Rekonstruktion, http://www.scanner. imagefact.de/de/sfm.html, (17.02.2017)

11. Tscharf, A.; Rumpler, M.; Mayer, G.; Bischof, H.: Zur Frage der Genauigkeit UAV-gestützter Tagebauvermessung, Schriftenreihe des Institutes für Markscheidewesen und Geodäsie, Heft 2014-1, S. $178-189$ 